\title{
Application of The Magnus and Fer Expansions to A Perturbative Study of The Time Dependent Jaynes Cummings Model
}

\author{
T. Das ${ }^{1}$ and A. Dasgupta ${ }^{2}$ \\ ${ }^{1}$ Department of Physics, Fakir Chand College, Diamond Harbour \\ ${ }^{2}$ Department of Physics, Indian Institute of Science and Educational Research, Kolkata \\ $1_{\text {tapas8791@gmail.com, }}$ \\ 2ananda.dasgupta@gmail.com
}

\begin{abstract}
We apply the Magnus and Fer perturbation methods to Jaynes Cummings Model (JCM) with linear time dependence and show that the perturbation methods yield results which are appreciably close to the results obtained by Wei-Norman method, leading to exact solution when the Hamiltonian under consideration is an element of su(2), thus making the perturbation methods important for Hamiltonians for which a method of exact solution is not available.
\end{abstract}

Keywords: JCM, Matrix, Evolution, Operator, Perturbation, Ramp, Sweep, Wei-Norman, Feynman-Dyson, Magnus, Fer.

Subject Classication: Quantum optics.

\section{Introduction}

Study of Jaynes Cummings Model[2] by several authors explored interesting behaviours of both the two-level atom and the radiation field of a coupled radiation-matter system $[6,7,8,9,10,11,12,13]$. The underlying $\mathrm{SU}(2)$ structure of the model[16] made it possible for application of the Wei-Norman method[3] for an exact solution. Dasgupta showed that the model is exactly solvable for a sech pulse[17] and for linear time dependence[19]. Limitations of exact solvability of the model with arbitrary time dependence lead us to follow perturbation methods as an alternative approach. The Hamiltonian of the model in the interaction picture is

$H(t)=2 \delta(\Delta) J_{3}+2 \Lambda(t) \sqrt{\chi(\Delta)} J_{1}(1)$

where is a constant operator

$\Delta=a^{\dagger} a+\frac{1+\sigma_{3}}{2}(2)$

and $c=a^{+} a, a^{+}$a being the number operator describing the strength of the field, $\left(a^{+}, a\right)$ being the creation and annihilation operator, respectively, describing the dynamics of the field and Ji are the generators of the SU(2) inherent in the Hamiltonian. We study the Hamiltonian (1) by assuming only one part of it to be linearly time dependent. The particular form of the Hamiltonian allows us to apply the methods of perturbation, assuming the time dependent part to be exactly solvable and the rest as a small perturbation. we expect that the procedures shall generate results with close proximity with those given by the exact solution, thereby, making it an alternative trick to approach time dependent Hamiltonians for which a method of exact solution is unavailable. 


\section{Perturbation Methods}

When a time dependent Hamiltonian $\mathrm{H}(\mathrm{t})$ does not commute with itself at dierent times, the usual approach for solution is the Feynman-Dyson method.

\subsection{Feynman-Dyson Formula}

The evolution operator is given by[21]

$$
U(t)=1+\sum_{k=1}^{\infty}(-i)^{k} \int_{t_{0}}^{t} d t_{1} \int_{t_{0}}^{t_{1}} d t_{2} \int_{t_{0}}^{t_{2}} d t_{3} \cdots \int_{t_{0}}^{t_{k-1}} d t_{k} H\left(t_{1}\right) H\left(t_{2}\right) \cdots H\left(t_{k}\right)
$$

Apart from the increasing difficulties in calculations in higher order, the formula suffers from a major drawback - the time evolution operator is not unitary at each order, which can be avoided by applying Magnus and Fer perturbation methods.

\subsection{Magnus Formula}

The evolution operator is expressed as exponential of an innite sum of anti-hermitian operators $[1,4,5,15,22]$

$$
U\left(t, t_{0}\right)=\exp \left[\sum_{n} A_{n}\left(t, t_{0}\right)\right]
$$

where the expansion terms An are given by

$$
\begin{gathered}
A_{1}(t)=-i \int_{t_{0}}^{t} d t_{1} H\left(t_{1}\right) \\
A_{2}(t)=\frac{1}{2 !} \int_{t_{0}}^{t} d t_{1} \int_{t_{0}}^{t_{1}} d t_{2}\left[H_{2}, H_{1}\right] \\
A_{3}(t)=\frac{i}{3 !} \int_{t_{0}}^{t} d t_{1} \int_{t_{0}}^{t_{1}} d t_{2} \int_{t_{0}}^{t_{2}} d t_{3}\left[\left[H_{3},\left[H_{2}, H_{1}\right]\right]+\left[\left[H_{3}, H_{2}\right], H_{1}\right]\right]
\end{gathered}
$$

and so on to the higer orders.

\subsection{Fer Formula}

According to Fer the time evolution operator is written as an innite product of exponential operators $[5,17,23]$

$$
U\left(t, t_{0}\right)=\prod_{j=1}^{\infty} \exp \left[S_{j}(t)\right](5)
$$

where

$$
\begin{gathered}
S_{1}(t)=-i \int_{t_{0}}^{t} H\left(t_{1}\right) d t_{1} \\
S_{2}(t)=\frac{1}{2 !} \int_{t_{0}}^{t} d t_{1} \int_{t_{0}}^{t_{1}} d t_{2}\left[H_{2}, H_{1}\right] \\
S_{3}(t)=\frac{i}{3 !} \int_{t_{0}}^{t} d t_{1} \int_{t_{0}}^{t_{1}} d t_{2} \int_{t_{0}}^{t_{2}} d t_{3}\left[\left[H_{3},\left[H_{2}, H_{1}\right]\right]+\left[H_{2},\left[H_{3}, H_{1}\right]\right]\right]
\end{gathered}
$$

and so on to the higer orders. 


\section{Perturbation method applied to JCM with linear time dependence}

\subsection{Interaction picture Hamiltonian}

We rename the picture in which the Hamiltonian (1) is written, to be 'Schrödinger Picture'. When the time dependence of the Hamiltonian is carried by only one term, it has the general form

$$
H(t)=H_{0}(t)+V(6)
$$

The time dependent part of the Hamiltonian is assumed to have an exact solution and the other time independent part is treated as a small perturbation to $\mathrm{HO}(\mathrm{t})$. With the initial time set at zero, the exact solution of the time dependent part is given by

$$
U_{0}(t)=\exp \left[i \int_{0}^{t} d t^{\prime} H_{0}\left(t^{\prime}\right)\right](7)
$$

We rid of the time dependent part by transforming (6) to 'Interaction Picture'

$$
H_{I}(t)=U_{0} V U_{0}^{+}(8)
$$

The form of this interaction picture Hamiltonian depends on the time dependence of $L(t)$ and involves the generators Ji of the SU(2), as evident from (1). This determines the evolution operator in interaction picture, at each order of a perturbation scheme.

\subsection{The evolution matrix}

We evaluate the time evolution operator at $k^{\text {th }}$ order of a perturbation scheme as a $2 \times 2$ matrix $\quad V(t)=v i j(k)$ using the algebra followed by Ji and its connection to the Pauli matrices through

$J_{i}=\sigma_{i} / 2(9)$

For Feynman-Dyson formula, straightforward substitution of (9) gives the evolution operator in matrix form.

For Magnus formula the sum inside the exponential of (4) is expressed in terms of the generators $\mathrm{Ji}$

$$
A(t)=-i\left[g_{1}(t) J_{1}+g_{2}(t) J_{2}+g_{3}(t) J_{3}\right](10)
$$

Using (9) the evolution operator is given in terms of the Pauli matrices

$$
U(t)=\cos f 1-i \frac{\sin f}{f}\left[f_{1}(t) \sigma_{1}+f_{2}(t) \sigma_{2}+f_{3}(t) \sigma_{3}\right]
$$

where $f(t)=g i(t) / 2$ and $f=\left(f 1^{2}+f 2^{2}+f 3^{2}\right)^{1 / 2}$. With $f_{ \pm}=f 1 \pm i f 2$, the evolution matrix becomes

$$
U(t)=\left(\begin{array}{cc}
\cos f-i f_{3} \frac{\sin f}{f} & -i f_{-} \frac{\sin f}{f} \\
-i f_{+} \frac{\sin f}{f} & \cos f+i f_{3} \frac{\sin f}{f}
\end{array}\right)
$$

Thus at the first order the evolution matrix is $\exp [A 1(t)]$, at the second order, it is $\exp [A 1(t)+A 2(t)]$ and so on The argument $\mathrm{Sj}(\mathrm{t})$ of the exponential in Fer formula (5), at each order, is given by 
$S_{j}(t)=-i\left[\alpha_{j}(t) \sigma_{1}+\beta_{j}(t) \sigma_{2}+\gamma_{j}(t) \sigma_{3}\right]$

by using (9). Thus, the evolution matrix at the $\mathrm{k}^{\text {th }}$ order is given by

$$
U_{k}(t)=\left(\begin{array}{cc}
\cos f_{k}-i \gamma_{k} \frac{\sin f_{k}}{f_{k}} & -i f_{k}^{(-)} \frac{\sin f_{k}}{f_{k}} \\
-i f_{k}^{(+)} \frac{\sin f_{k}}{f_{k}} & \cos f_{k}+i \gamma_{k} \frac{\sin f_{k}}{f_{k}}
\end{array}\right.
$$

with

$f_{k}^{2}=\alpha_{k}^{2}+\beta_{k}^{2}+\gamma_{k}^{2}, \quad$ and $\quad f_{k}^{ \pm}=\alpha_{k} \pm i \beta_{k}$

At the first order, the evolution operator is $U(t)=U 1(t)$, to the second order it is $U(t)=U 1(t) U 2(t)$ and so on . Thus, one may calculate the evolution operator in explicit analytic form to any desired order.

\subsection{Linear Ramp}

We call the linear time dependence a linear ramp when the detuning parameter is linearly time dependent, $d(D)=b t$ and the interaction parameter is time independent, $L(t)=10$, so that the Hamiltonian (1) becomes

$$
H(t)=2 \beta t J_{3}+2 \lambda_{0} \sqrt{\chi} J_{1}
$$

Evolution operator for the time dependent part of (16) is

$$
U_{0}(t)=\exp \left[i \beta t^{2} J_{3}\right]
$$

The 'Interaction Picture' Hamiltonian is given by

$$
H_{I}(t)=2 \delta\left[\cos \left(\beta t^{2}\right) J_{1}-\sin \left(\beta t^{2}\right) J_{2}\right]
$$

\subsubsection{Feynman-Dyson}

Up to first order, the evolution operator is given by

$$
V^{(1)}=\left(\begin{array}{cc}
1 & \delta\left(I_{2}-i I_{1}\right) \\
-\delta\left(I_{2}+i I_{1}\right) & 1
\end{array}\right)
$$

Up to second order,

$$
V^{(2)}=\left(\begin{array}{cc}
1-\delta^{2}\left[\left(I_{3}+I_{6}\right)+i\left(I_{5}-I_{4}\right)\right] & \delta\left(I_{2}-i I_{1}\right) \\
-\delta\left(I_{2}+i I_{1}\right) & 1-\delta^{2}\left[\left(I_{3}+I_{6}\right)-i\left(I_{5}-I_{4}\right)\right]
\end{array}\right)
$$

Up to third order,

$$
V^{(3)}=\left(\begin{array}{cc}
1-\delta^{2}\left[\left(I_{3}+I_{6}\right)+i\left(I_{5}-I_{4}\right)\right] & \delta\left(I_{2}-i I_{1}\right)+i \delta^{3}\left(k_{1}+i k_{2}\right) \\
-\delta\left(I_{2}+i I_{1}\right)+i \delta^{3}\left(k_{1}-i k_{2}\right) & 1-\delta^{2}\left[\left(I_{3}+I_{6}\right)-i\left(I_{5}-I_{4}\right)\right]
\end{array}\right)
$$

with 


$$
\begin{gathered}
k_{1}=I_{7}+I_{10}-I_{12}+I_{13} \\
k_{2}=I_{8}-I_{9}+I_{11}+I_{14}
\end{gathered}
$$

\subsubsection{Magnus}

As mentioned in subsection 2.2, the functions $\mathrm{fk}(\mathrm{t})$ occurring in (4) determines the matrix elements of the evolution operator

at each

order.

At the first order,

$f_{1}(t)=2 \delta I_{1}, \quad f_{2}(t)=-2 \delta I_{2}, \quad f_{3}(t)=0(23)$

At the second order,

$f_{1}=2 \delta I_{1}, \quad f_{2}=-2 \delta I_{2}, \quad f_{3}=2 \delta^{2}\left(I_{5}-I_{4}\right)(24)$

At the third order,

$f_{1}=2 \delta I_{1}-\frac{4}{3} \delta^{3}\left(I_{10}-2 I_{12}+I_{13}\right), \quad f_{2}=-2 \delta I_{2}+\frac{4}{3} \delta^{3}\left(I_{8}-2 I_{9}+I_{11}\right), \quad f_{3}=2 \delta^{2}\left(I_{5}-I_{4}\right)$

\subsubsection{Fer}

As mentioned in subsection 2.3, the functions $\mathrm{fk}(\mathrm{t})$ occurring in (13) determines the matrix elements of the evolution operator

at

each

order.

At the first order,

$f_{1}(t)=2 \delta I_{1}, \quad f_{2}(t)=-2 \delta I_{2}, \quad f_{3}(t)=0(26)$

At the second order,

$f_{1}=0, \quad f_{2}=0, \quad f_{3}=2 \delta^{2}\left(I_{5}-I_{4}\right)$

At the third order,

$f_{1}(t)=\frac{4}{3} \delta^{3}\left(-2 I_{10}+I_{12}+I_{13}\right), \quad f_{2}(t)=\frac{4}{3} \delta^{3}\left(-I_{8}-I_{9}+2 I_{11}\right), \quad f_{3}(t)=0 \quad(28)$

\subsection{Linear sweep}

Calling the case with time independent detuning $d(D)=d$ and linearly time dependent interaction parameter $L(t)=10 t$ a linear sweep, the Hamiltonian (1) becomes

$H(t)=2 \delta J_{3}+2 \lambda_{0} t \sqrt{\chi} J_{1}$

Evolution operator for the time dependent part of (29) is

$U_{0}(t)=\exp \left[i \beta t^{2} J_{1}\right]$

The 'Interaction Picture' Hamiltonian is given by

$H_{I}(t)=2 \delta\left[\sin \left(\beta t^{2}\right) J_{2}+\cos \left(\beta t^{2}\right) J_{3}\right]$ 


\subsubsection{Feynman-Dyson}

Up to first order,

$V^{(1)}=\left(\begin{array}{cc}1-i \delta I_{1} & -\delta I_{2} \\ \delta I_{2} & 1+i \delta I_{1}\end{array}\right)$

Up to second order,

$V^{(2)}=\left(\begin{array}{cc}1-i \delta I_{1}-\delta^{2}\left(I_{3}+I_{6}\right) & -\delta I_{2}+i \delta^{2}\left(I_{4}-I_{5}\right) \\ \delta I_{2}+i \delta^{2}\left(I_{4}-I_{5}\right) & 1+i \delta I_{1}-\delta^{2}\left(I_{3}+I_{6}\right)\end{array}\right)$

Up to third order,

$V^{(3)}=\left(\begin{array}{cc}1-i \delta I_{1}-\delta^{2}\left(I_{3}+I_{6}\right)+i \delta^{3} k_{1} & -\delta I_{2}+i \delta^{2}\left(I_{4}-I_{5}\right)+\delta^{3} k_{2} \\ \delta I_{2}+i \delta^{2}\left(I_{4}-I_{5}\right)-\delta^{3} k_{2} & 1+i \delta I_{1}-\delta^{2}\left(I_{3}+I_{6}\right)-i \delta^{3} k_{1}\end{array}\right)$

with

$k_{1}=I_{7}+I_{10}-I_{12}+I_{13}$

$k_{2}=I_{8}-I_{9}+I_{11}+I_{14}$

\subsubsection{Magnus}

As mentioned in subsection 2.2, the functions $\mathrm{fk}(\mathrm{t})$ occurring in (10) determines the matrix elements of the evolution operator at each order.

First order,

$f_{1}(t)=0, \quad f_{2}(t)=2 \delta I_{2}, \quad f_{3}(t)=2 \delta I_{1}(35)$

Second order,

$f_{1}=2 \delta^{2}\left(I_{5}-I_{4}\right), \quad f_{2}=2 \delta I_{2}, \quad f_{3}=2 \delta I_{1}(36)$

Third order,

$f_{1}=2 \delta^{2}\left(I_{5}-I_{4}\right), \quad f_{2}=2 \delta I_{2}+\frac{4}{3} \delta^{3}\left(I_{8}-2 I_{9}+I_{11}\right), \quad f_{3}=2 \delta I_{1}-\frac{4}{3} \delta^{3}\left(I_{10}-2 I_{12}+I_{13}\right)$

\subsubsection{Fer}

As mentioned in subsection 2.3, the functions $\mathrm{fk}(\mathrm{t})$ occurring in (13) determines the matrix elements of the evolution operator

at

each

order.

First order,

$f_{1}(t)=0, \quad f_{2}(t)=2 \delta I_{2}, \quad f_{3}(t)=2 \delta I_{1}(38)$

Second order, 
$f_{1}=2 \delta^{2}\left(I_{5}-I_{4}\right), \quad f_{2}=2 \delta I_{2}, \quad f_{3}=2 \delta I_{1}(39)$

Third order,

$f_{1}=0, \quad f_{2}=\frac{4}{3} \delta^{3}\left(I_{8}+I_{9}-2 I_{11}\right), \quad f_{3}=\frac{4}{3} \delta^{3}\left(-2 I_{10}+I_{12}+I_{13}\right)(40)$

The time dependent integrals $I k(t)$ are presented in the appendix and are computed using python scripts.

\section{Results and Discussion}

\subsection{Linear ramp}

Fig.1 and Fig.2 show, respectively, that the time evolution of inversion and Mandel Q parameter determined by Feynman-Dyson(FD) formula at second and third orders, are much better than those at the first order. Similar feature is evident for squeezing - FD formula, even at first order, gives results which are appreciably close to the result of Wei-Norman(WN) method(Fig.2). Also, the figures indicate an order-by-order improvement of the results. This order-by-order improvement of the results are studied with a greater detail by computing the logarithmic difference of inversions obtained from WN method and FD formula(Fig.4). As shown, a larger negative value of the logarithmic difference shows an improvement of the result at higher order of the perturbation method. Study of the physical quantities with Magnus and Fer perturbation formulae show similar features(Fig.7,8,9 and Fig.13,14,15), along with a clear indication that Magnus and Fer methods yield even better results than FD formula(Fig.10,11,12 and Fig.16,17,18).

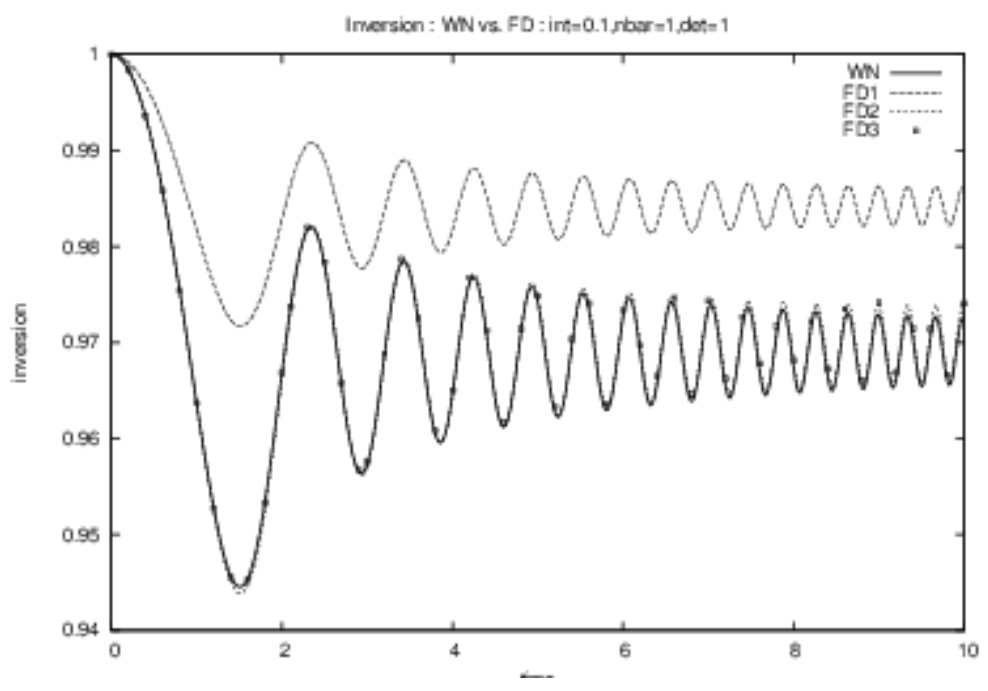

Figure 1: Ramp : Comparing WN and FD : Variation of inversion. 


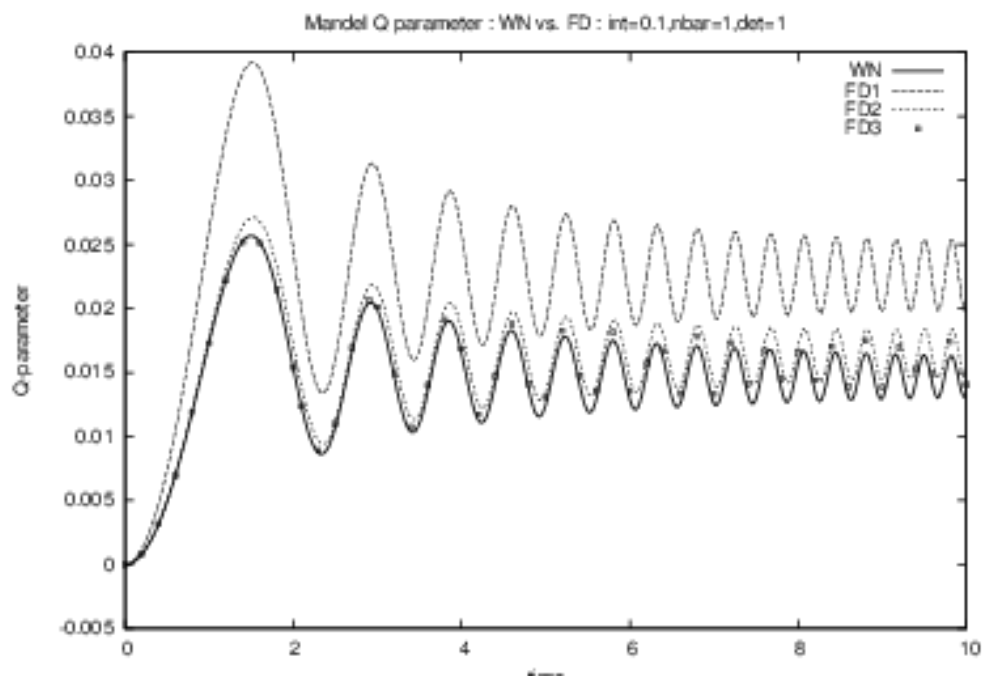

Figure 2: Ramp : Comparing WN and FD : Variation of Mandel Q parameter.

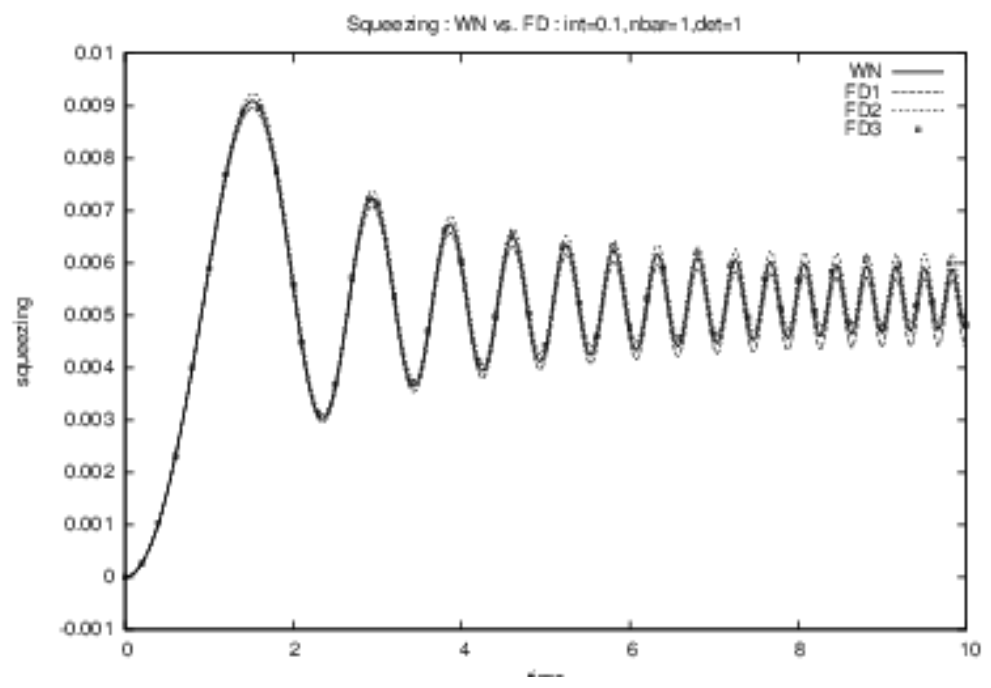

Figure 3: Ramp : Comparing WN and FD : Variation of squeezing.

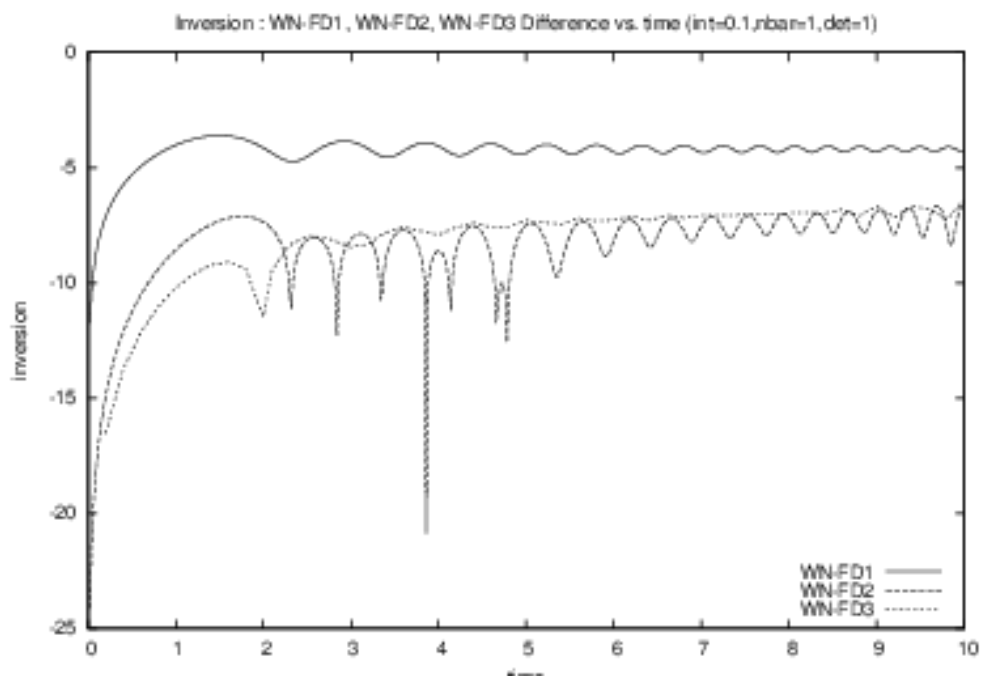

Figure 4: Ramp : Comparing WN and FD : Variation of inversion : Lorgarithmic Difference. 


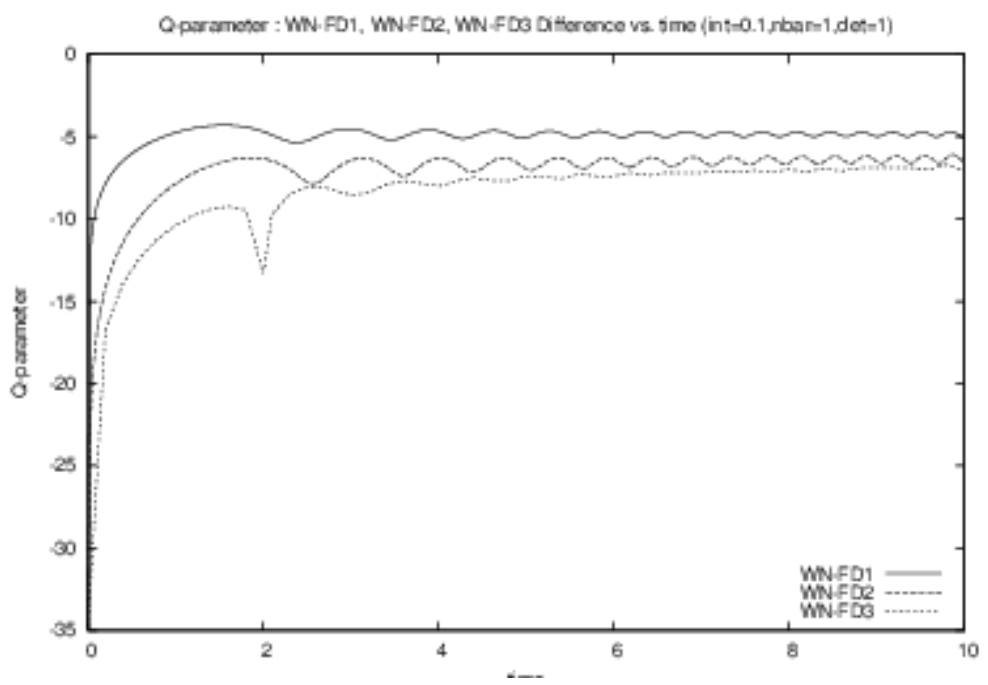

Figure 5: Ramp : Comparing WN and FD : Variation of Mandel Q parameter : Lorgarithmic Difference.

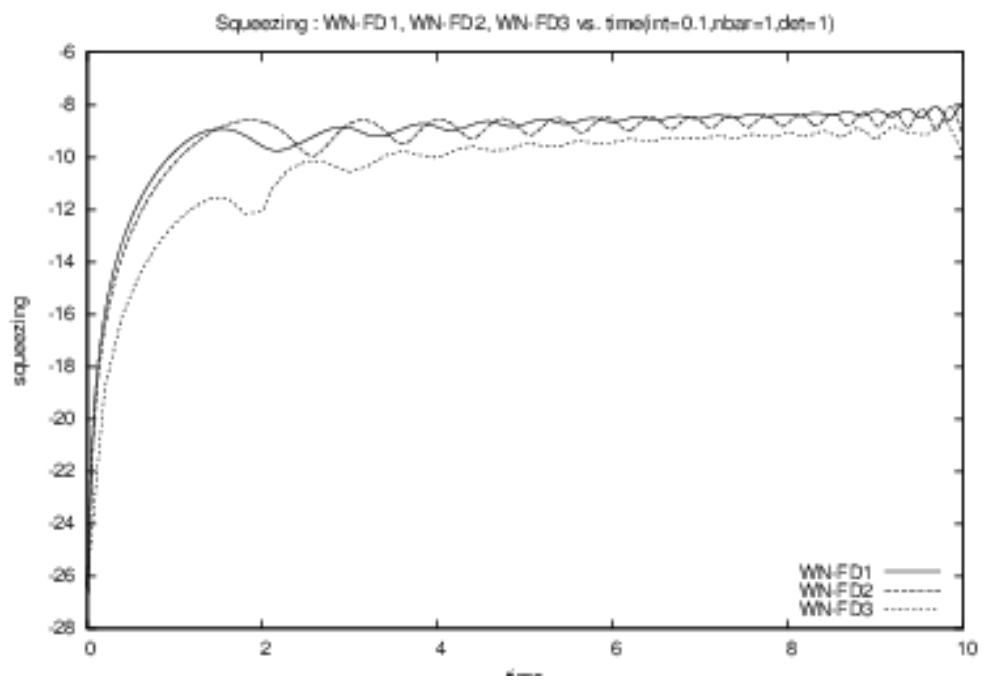

Figure 6: Ramp : Comparing WN and FD : Variation of squeezing : Lorgarithmic Difference.

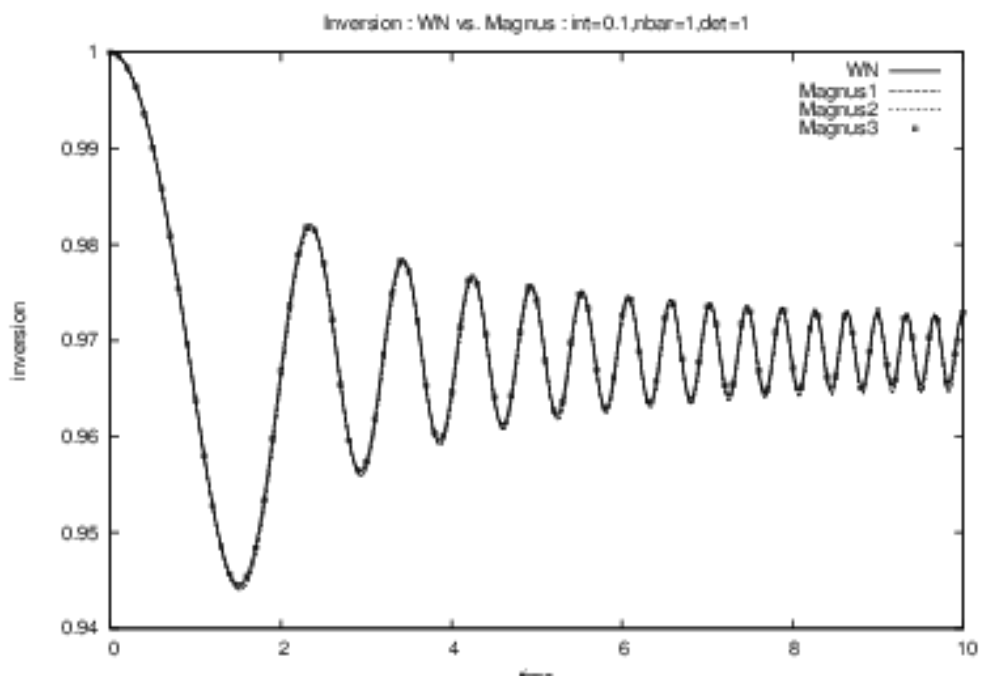

Figure 7: Ramp : Comparing WN and Magnus : Variation of inversion. 


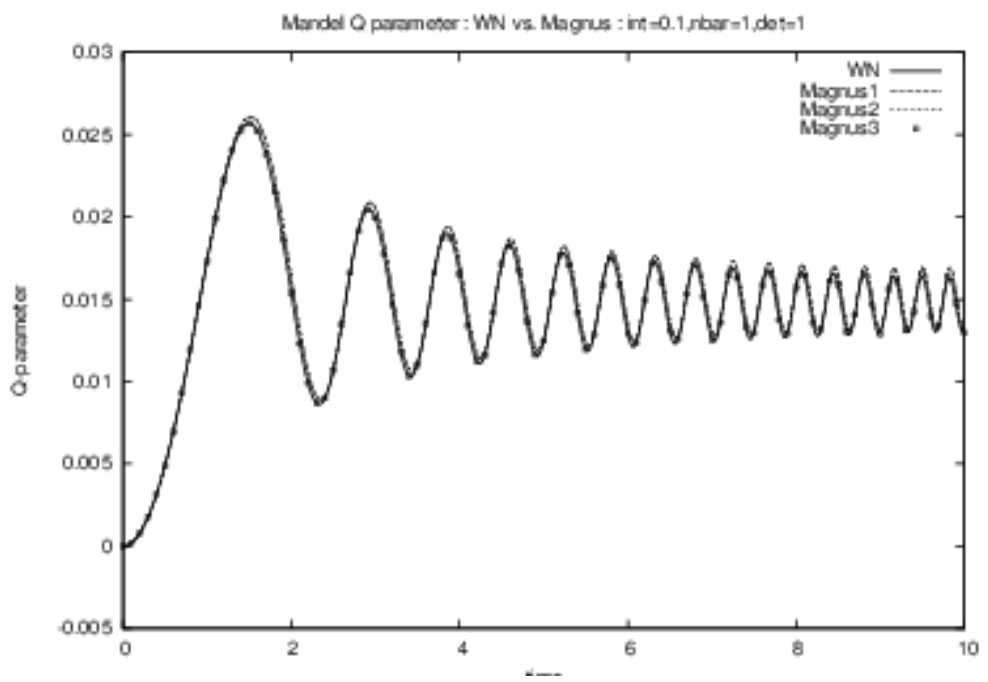

Figure 8: Ramp : Comparing WN and Magnus : Variation of Mandel Q parameter.

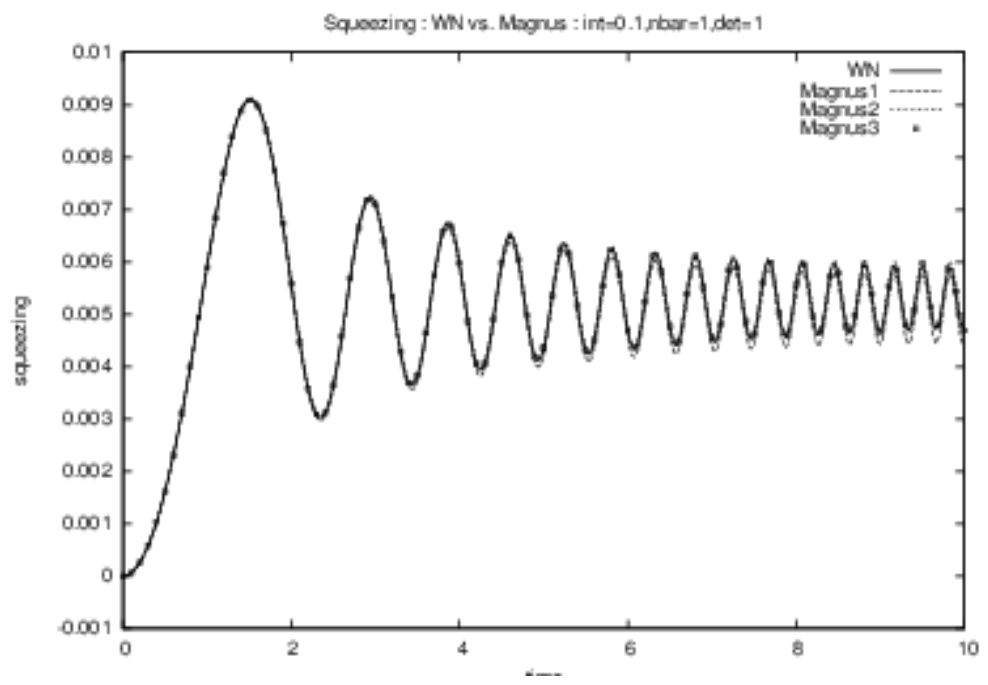

Figure 9: Ramp : Comparing WN and Magnus : Variation of squeezing.

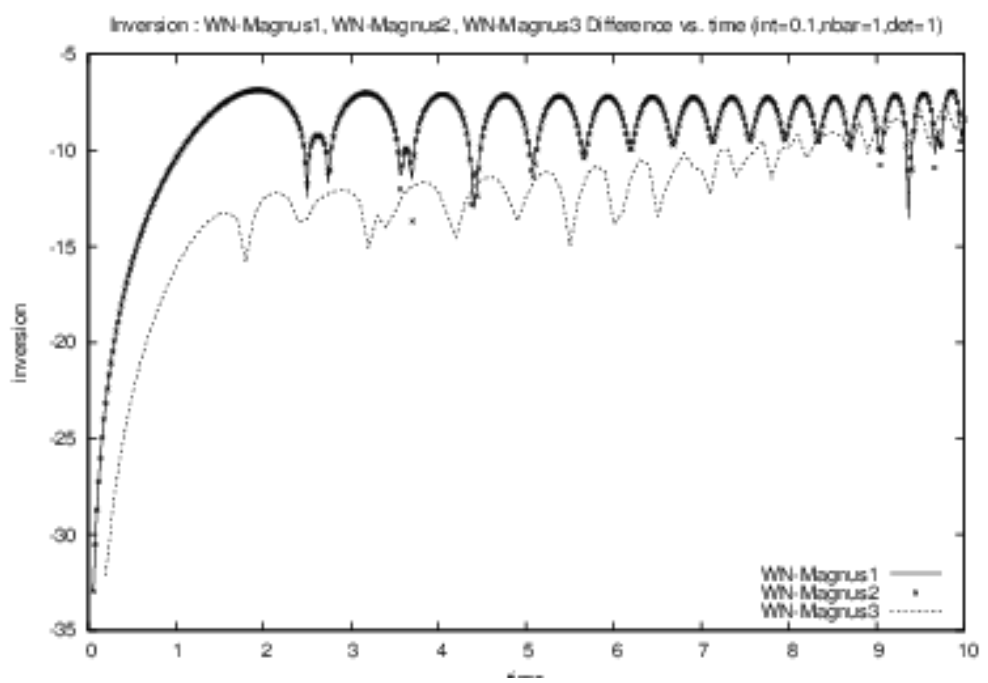

Figure 10: Ramp : Comparing WN and Magnus : Variation of inversion : Lorgarithmic Difference. 


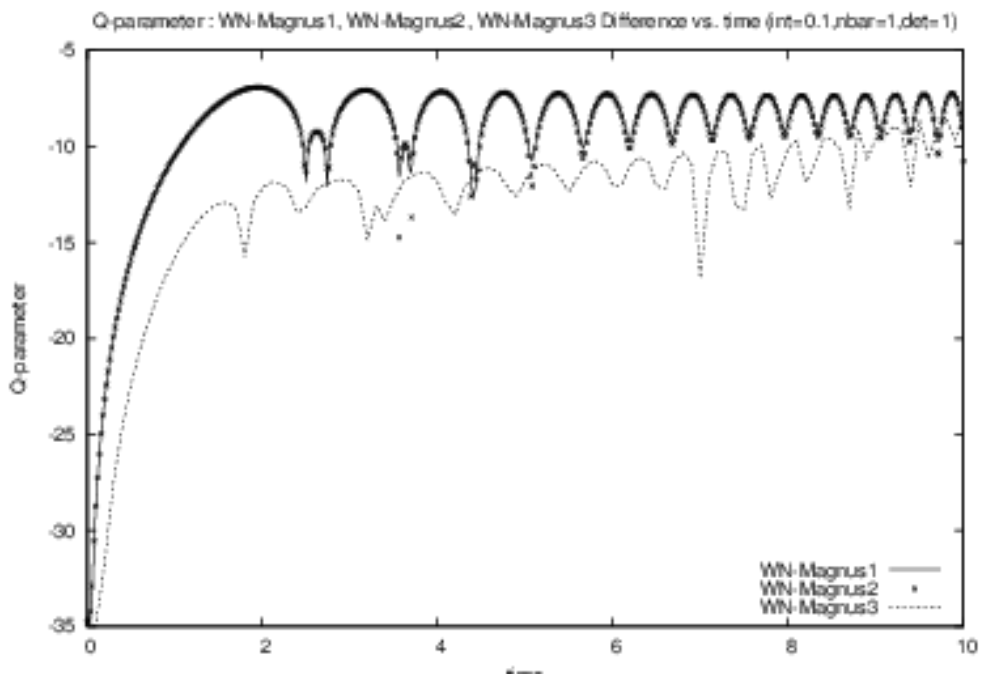

Figure 11: Ramp : Comparing WN and Magnus : Variation of Mandel Q parameter : Lorgarithmic Difference.

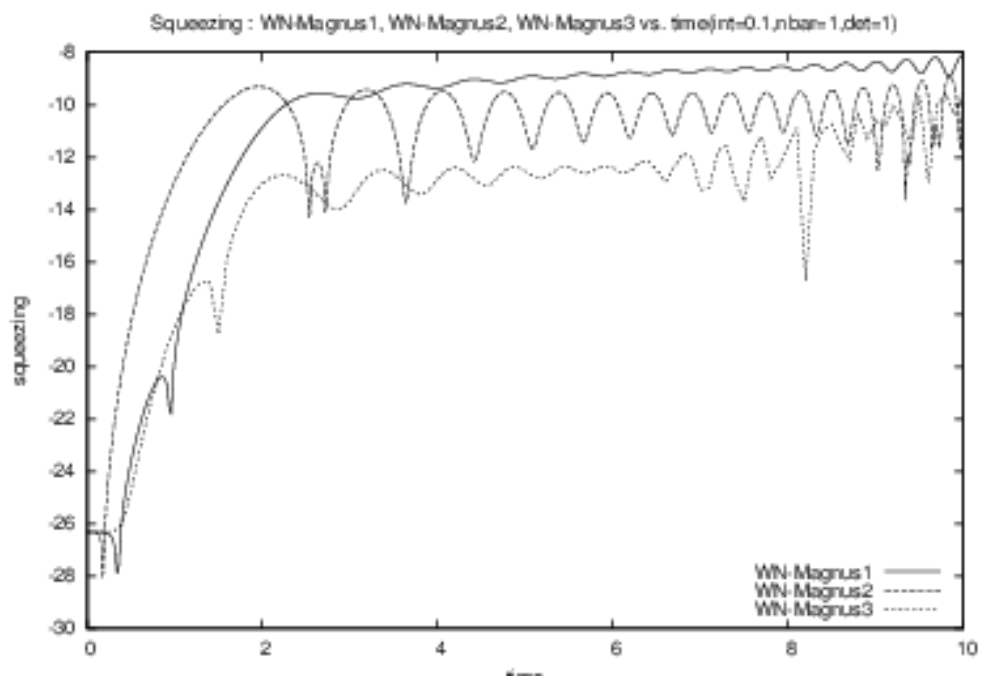

Figure 12: Ramp : Comparing WN and Magnus : Variation of squeezing : Lorgarithmic Difference.

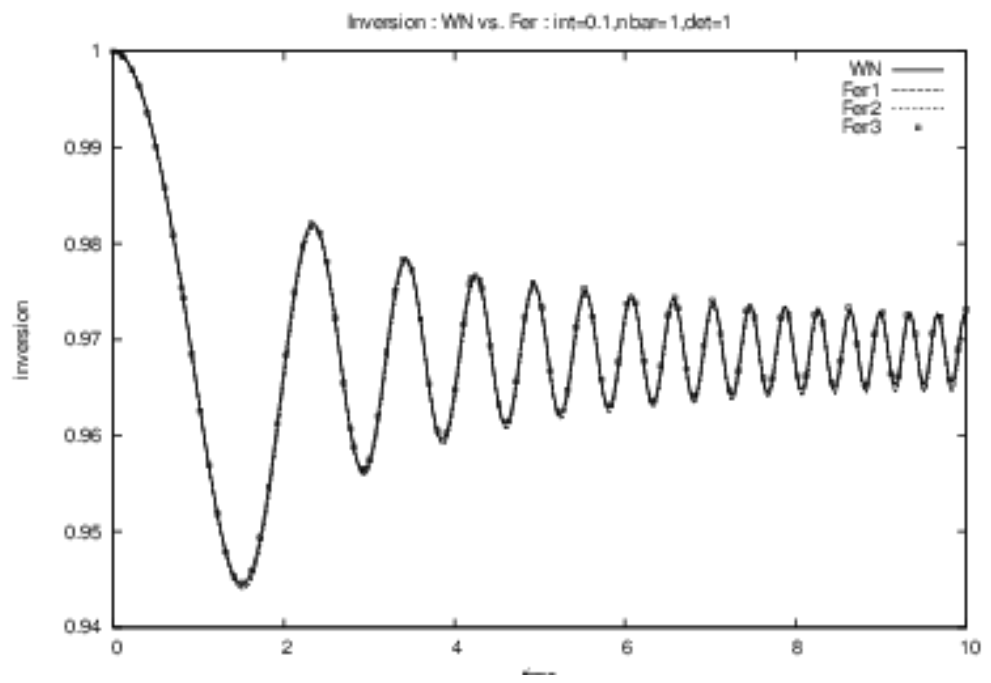

Figure 13: Ramp : Comparing WN and Fer : Variation of inversion. 


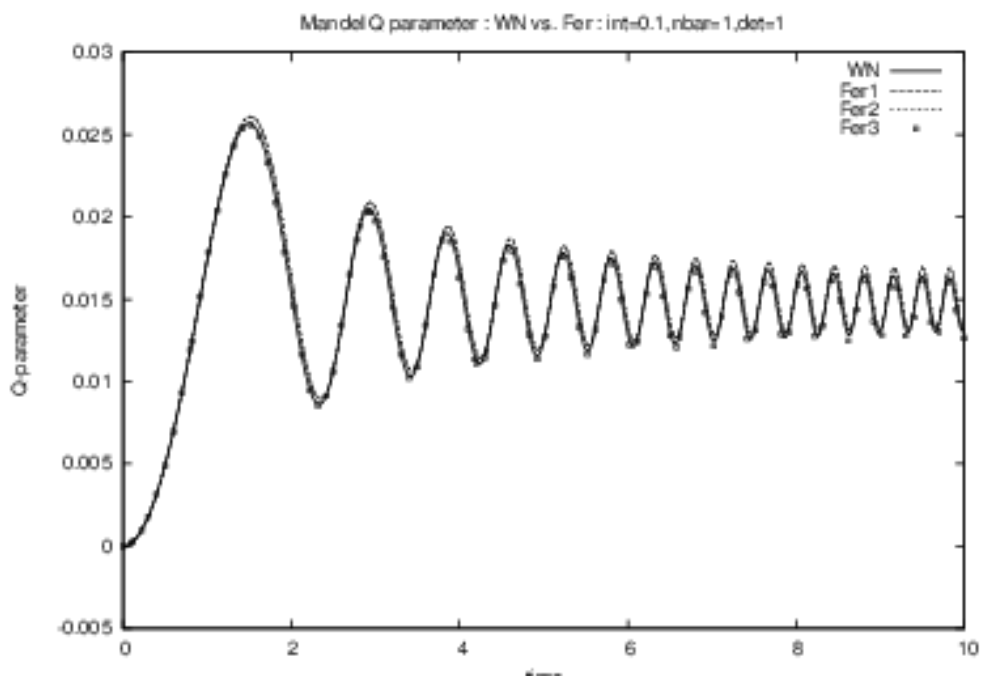

Figure 14: Ramp : Comparing WN and Fer : Variation of Mandel Q parameter.

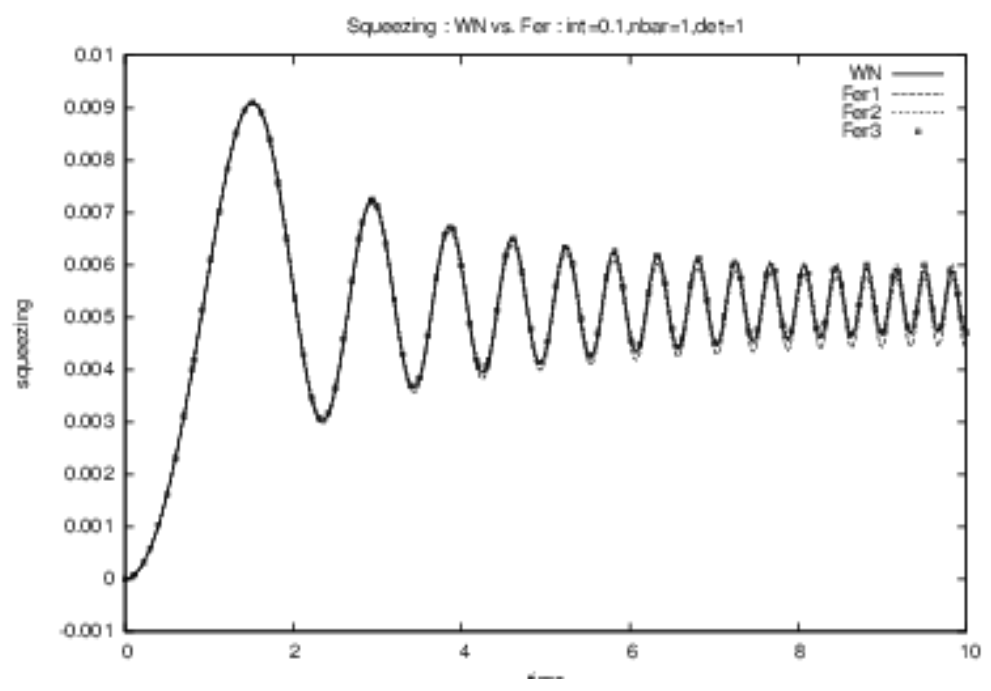

Figure 15: Ramp : Comparing WN and Fer : Variation of squeezing.

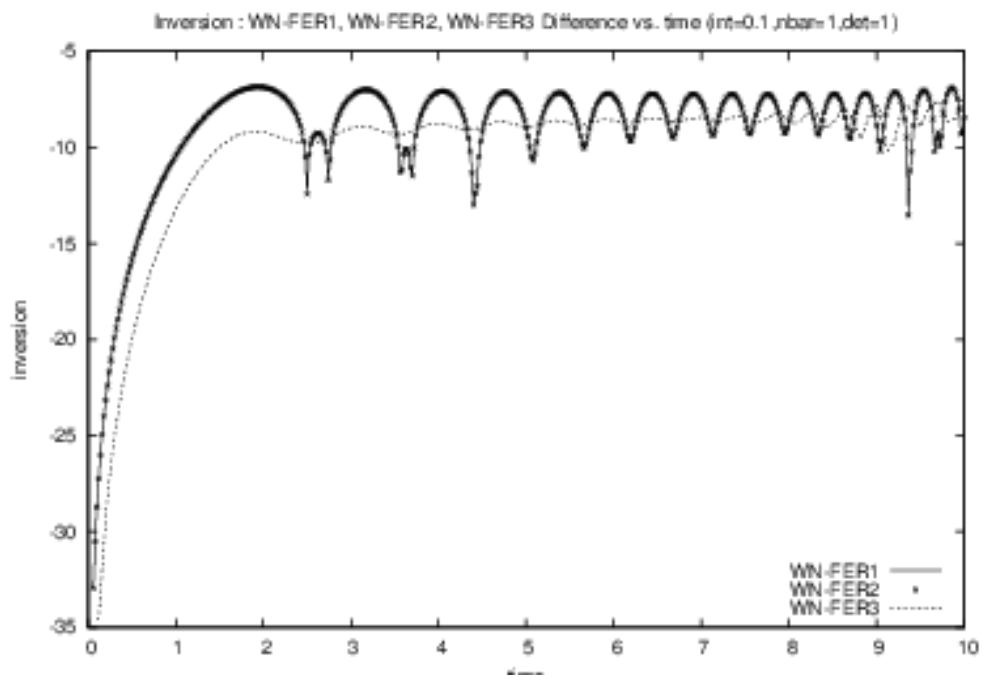

Figure 16: Ramp : Comparing WN and Fer : Variation of inversion : Lorgarithmic Difference. 


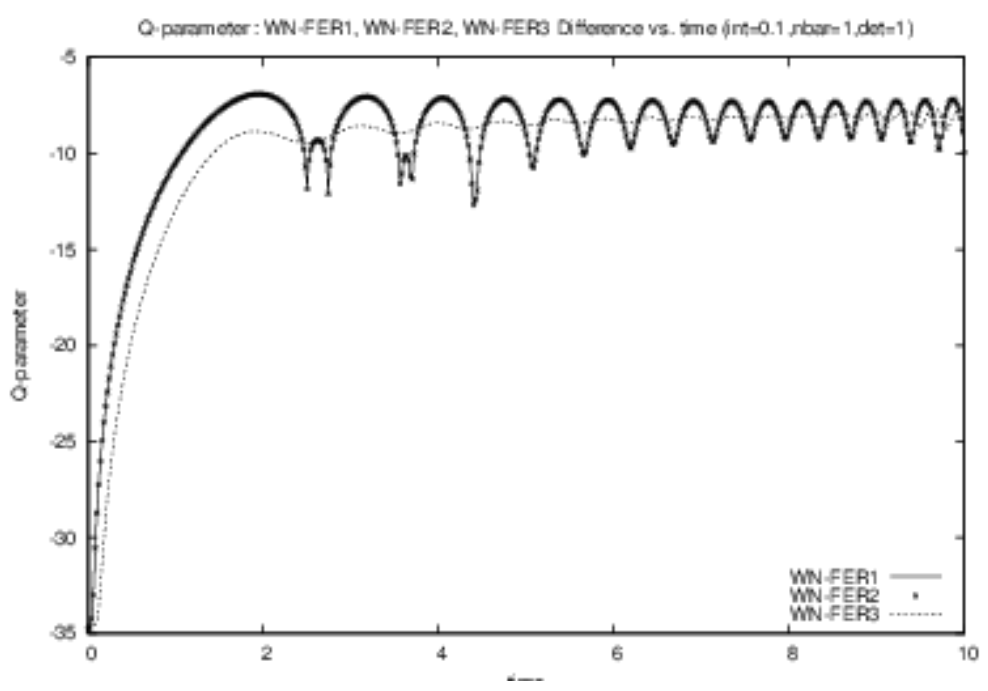

Figure 17: Ramp : Comparing WN and Fer : Variation of Mandel Q parameter : Lorgarithmic Difference.

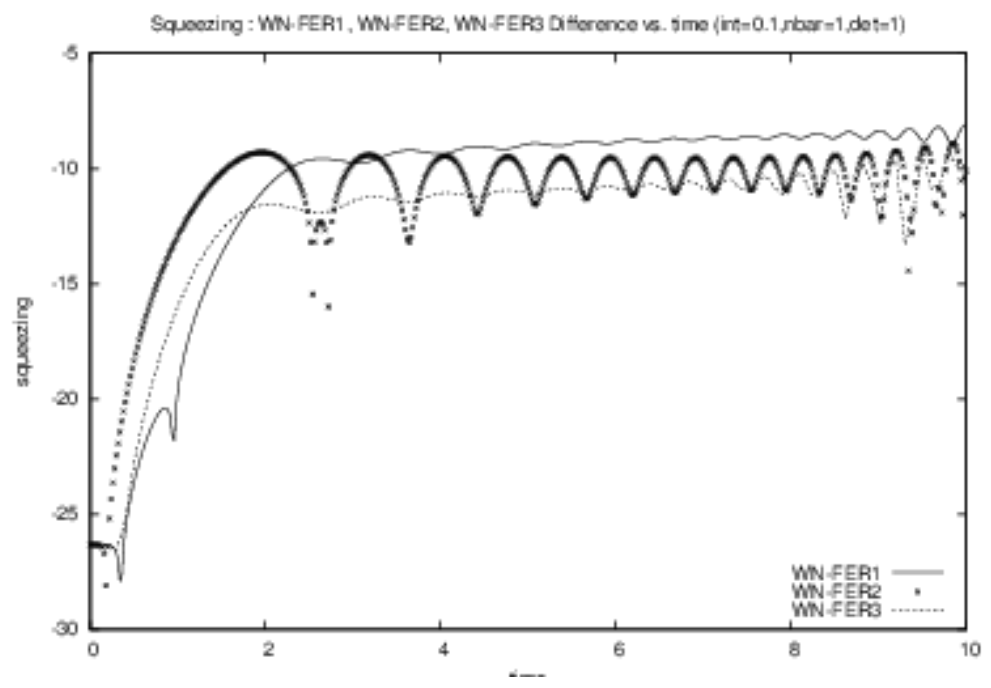

Figure 18: Ramp : Comparing WN and Fer : Variation of squeezing : Lorgarithmic Difference.

\subsection{Linear sweep}

As for the case of linear ramp, the closeness of the results yielded by the perturbation methods to those generated by Wei-Norman method is evident from the figures $19,20,21,25,26,27,31,32$ and 33 . The gures $22,23,24,28,29,30,34,35$ and 36 show the order-by-order improvement of a perturbation scheme. 


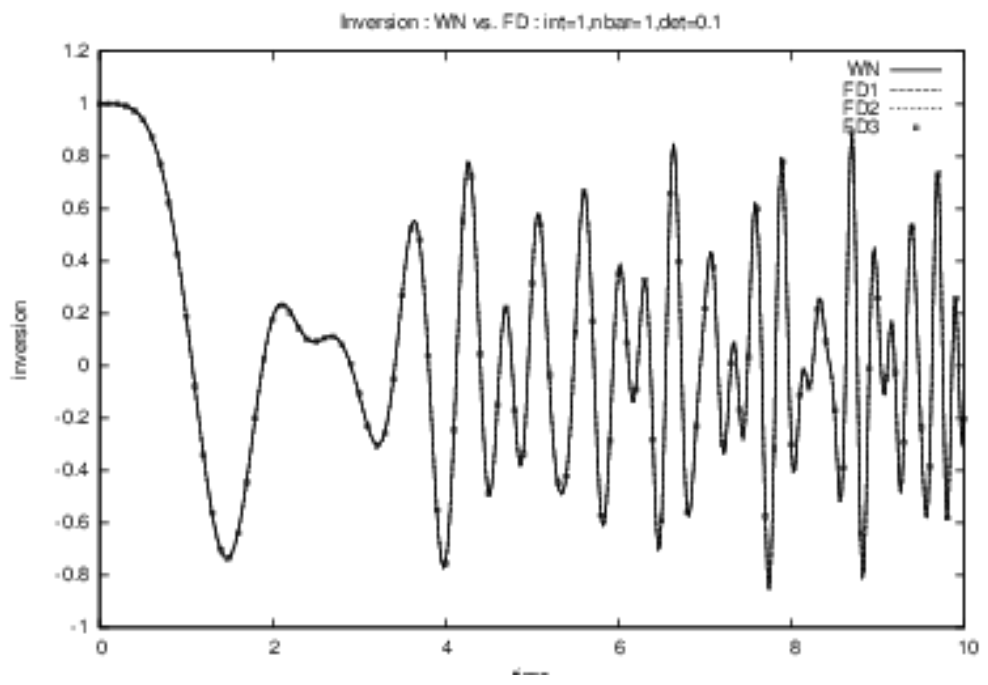

Figure 19: Sweep : Comparing WN and FD : Variation of inversion.

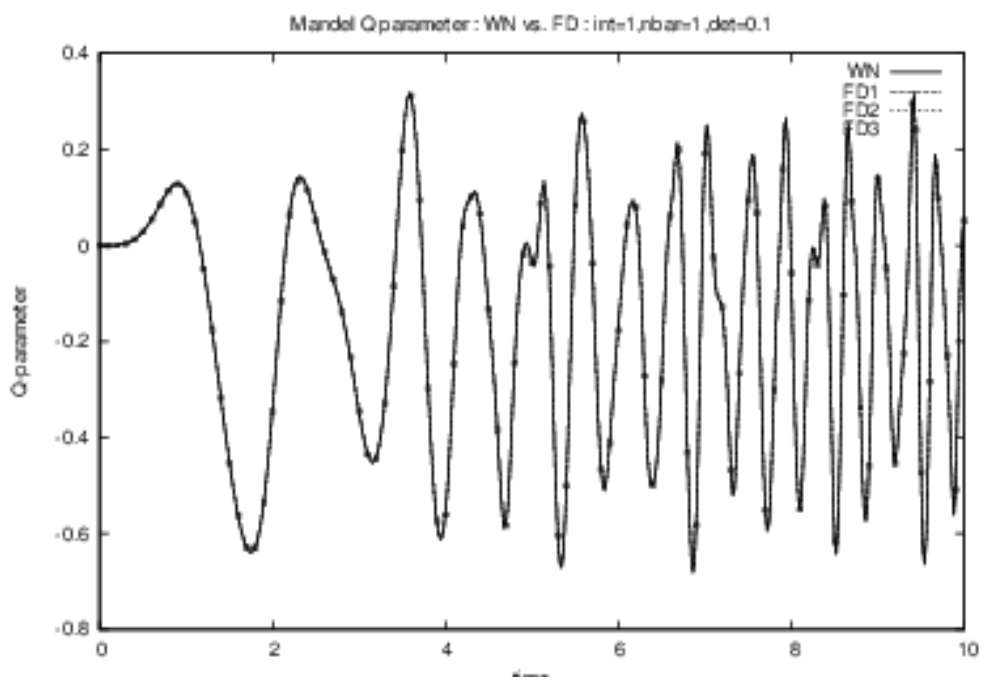

Figure 20: Sweep : Comparing WN and FD : Variation of Mandel Q parameter.

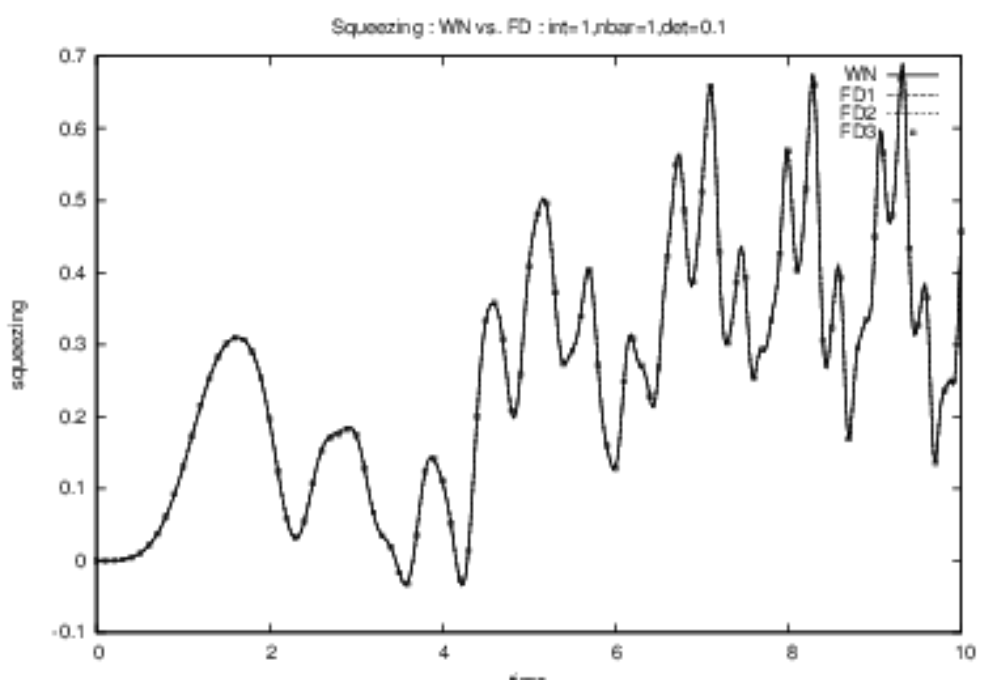

Figure 21: Sweep : Comparing WN and FD : Variation of squeezing. 


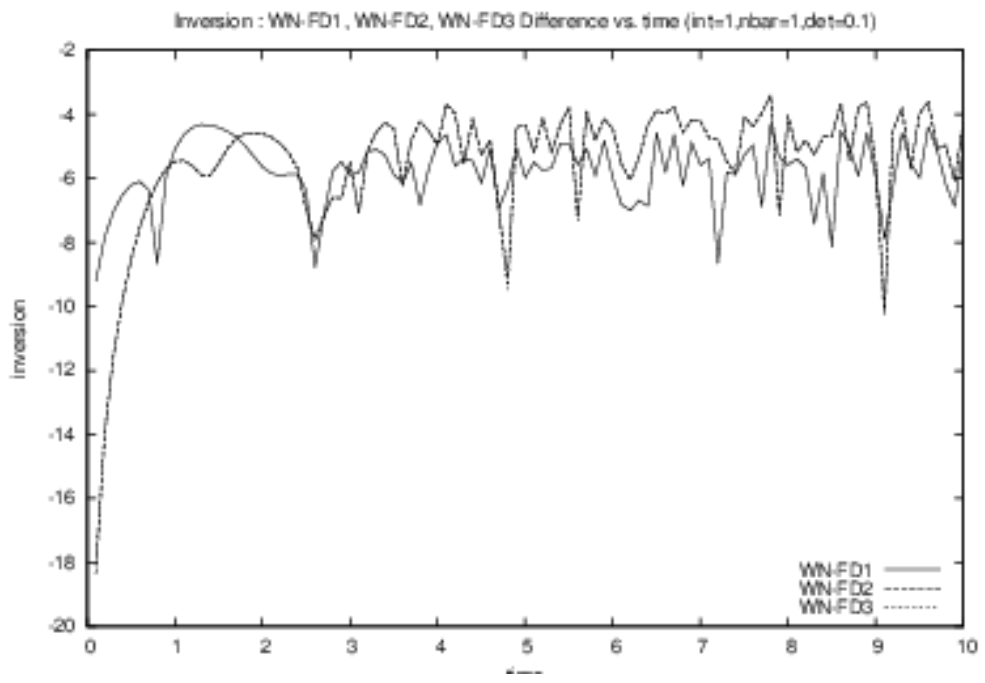

Figure 22: Sweep : Comparing WN and FD : Variation of inversion : Lorgarithmic Difference.

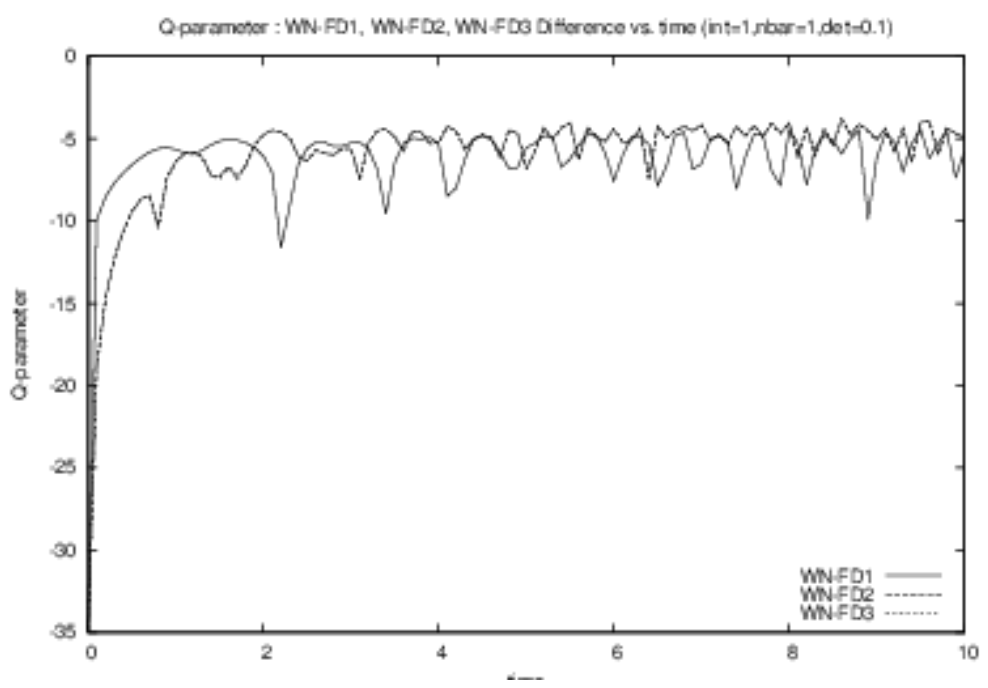

Figure 23: Sweep : Comparing WN and FD : Variation of Mandel Q parameter : Lorgarithmic Difference.

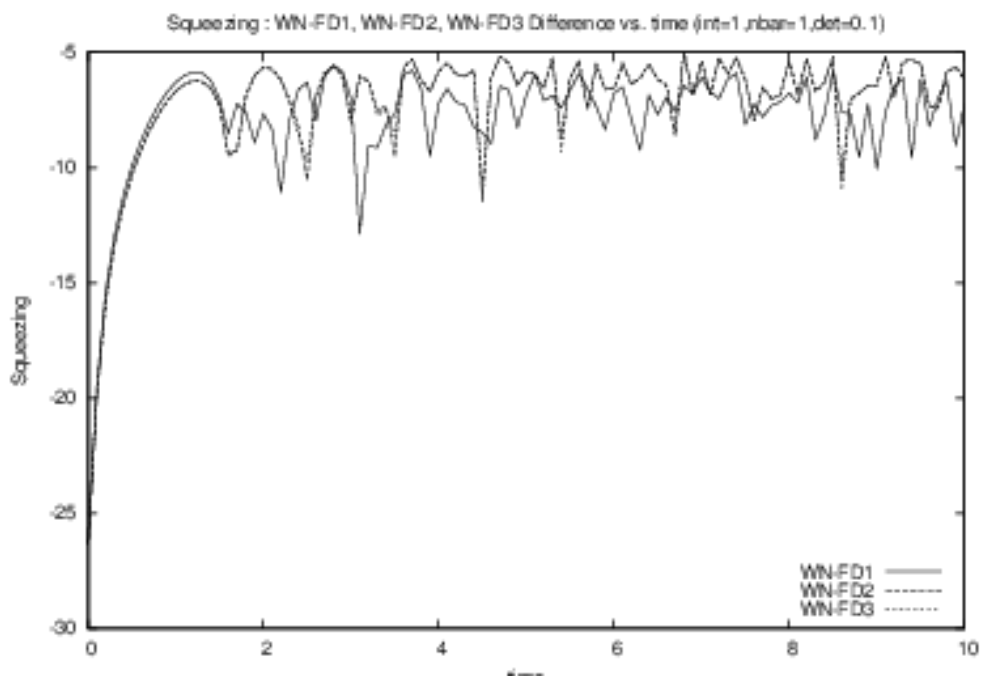

Figure 24: Sweep : Comparing WN and FD : Variation of squeezing : Lorgarithmic Dffierence. 


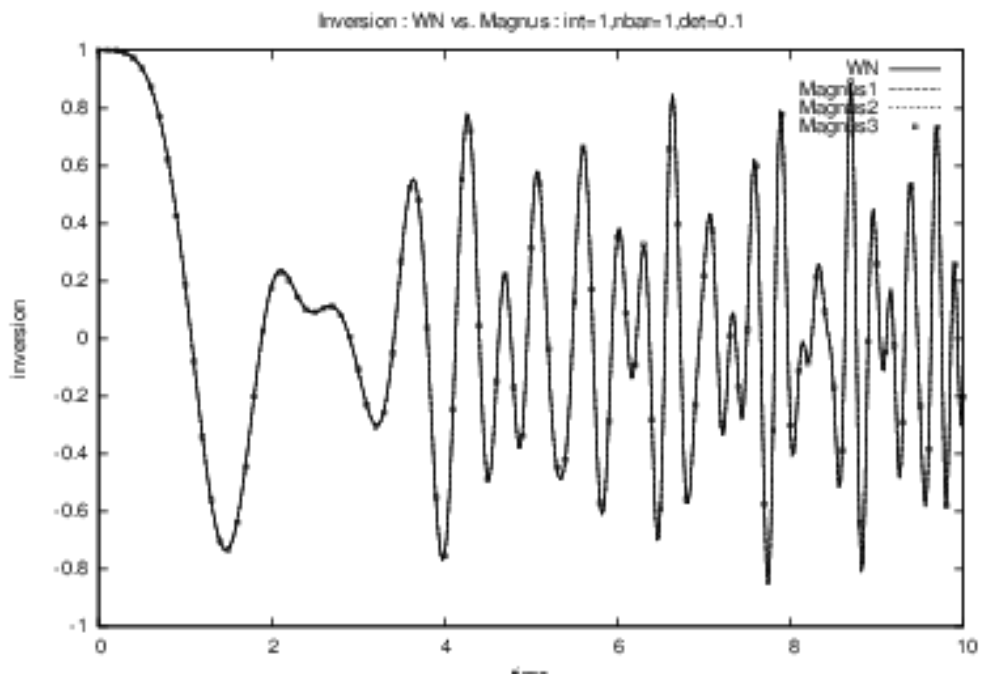

Figure 25: Sweep : Comparing WN and Magnus : Variation of inversion.

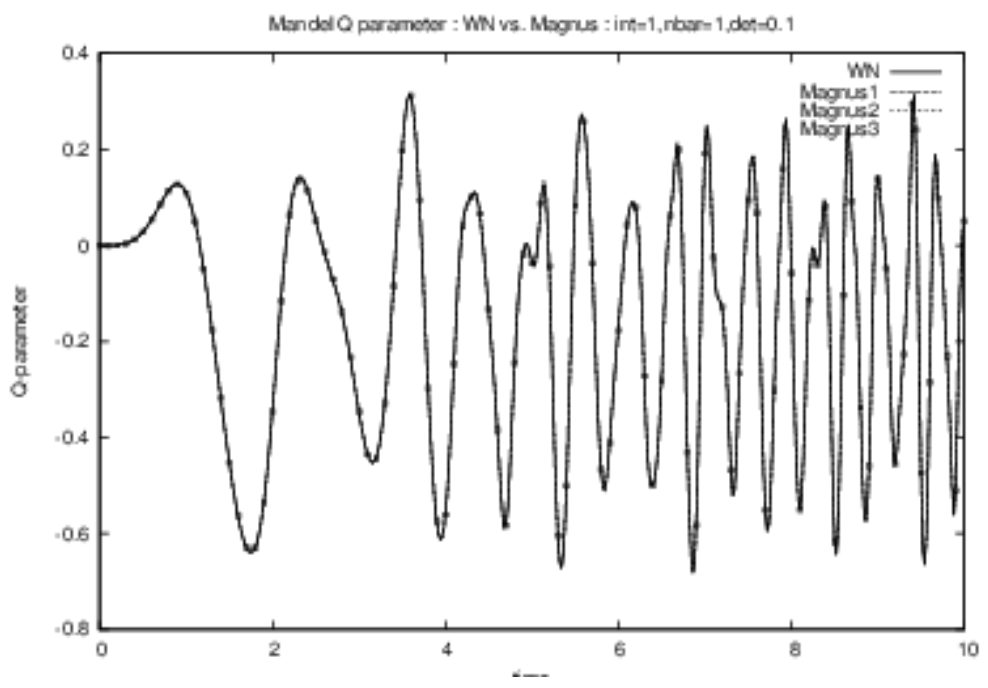

Figure 26: Sweep : Comparing WN and Magnus : Variation of Mandel Q parameter.

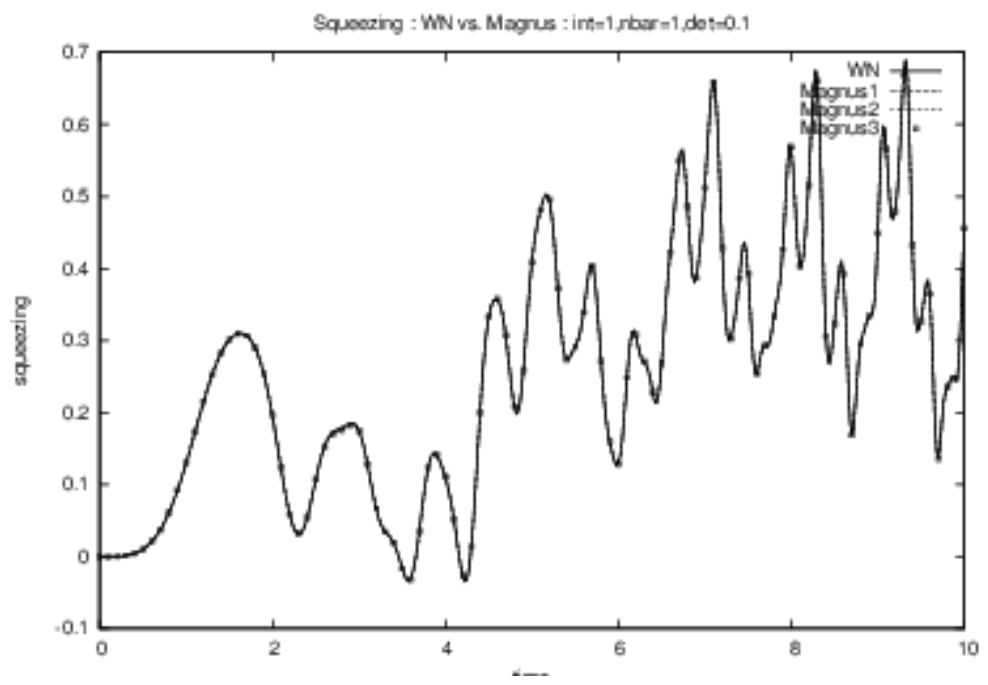

Figure 27: Sweep : Comparing WN and Magnus : Variation of squeezing. 


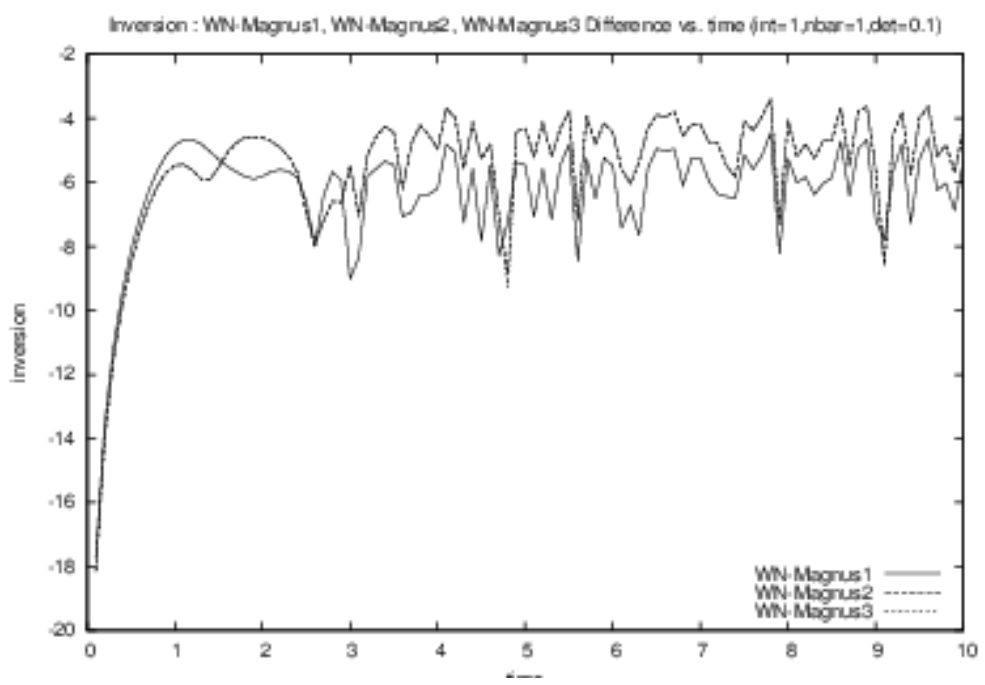

Figure 28: Sweep : Comparing WN and Magnus : Variation of inversion : Lorgarithmic Difference.

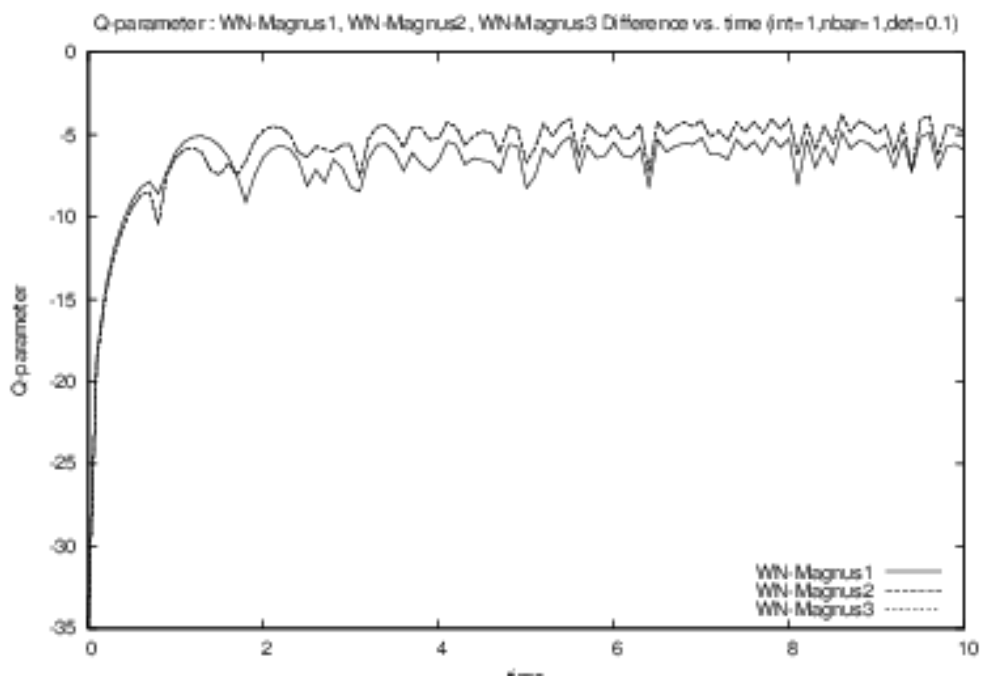

Figure 29: Sweep : Comparing WN and Magnus : Variation of Mandel Q parameter : Lorgarithmic Difference.

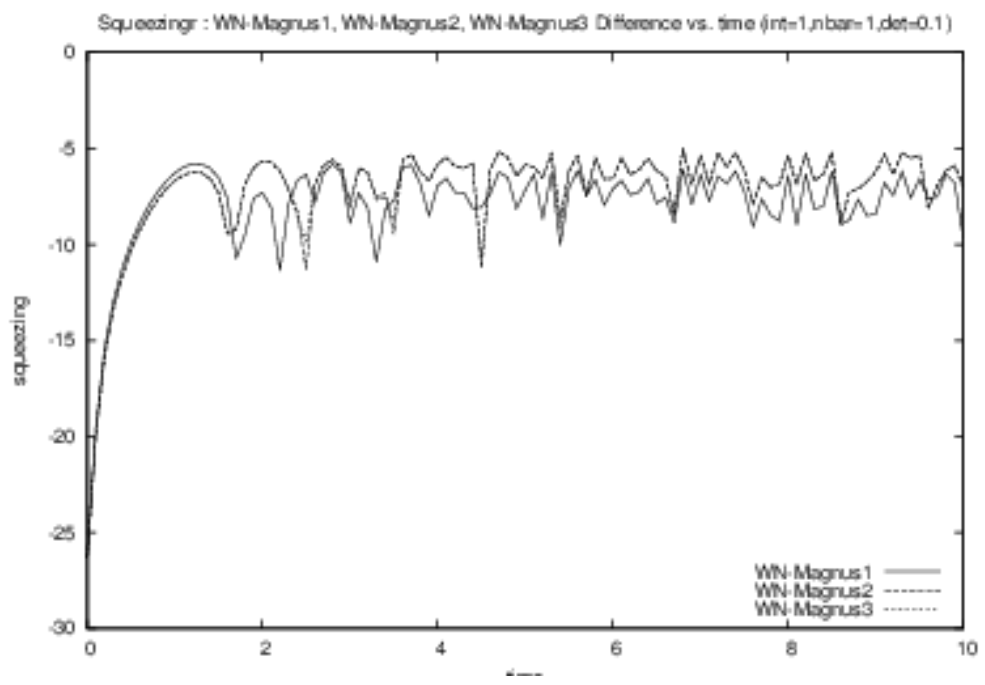

Figure 30: Sweep : Comparing WN and Magnus : Variation of squeezing : Lorgarithmic Difference. 


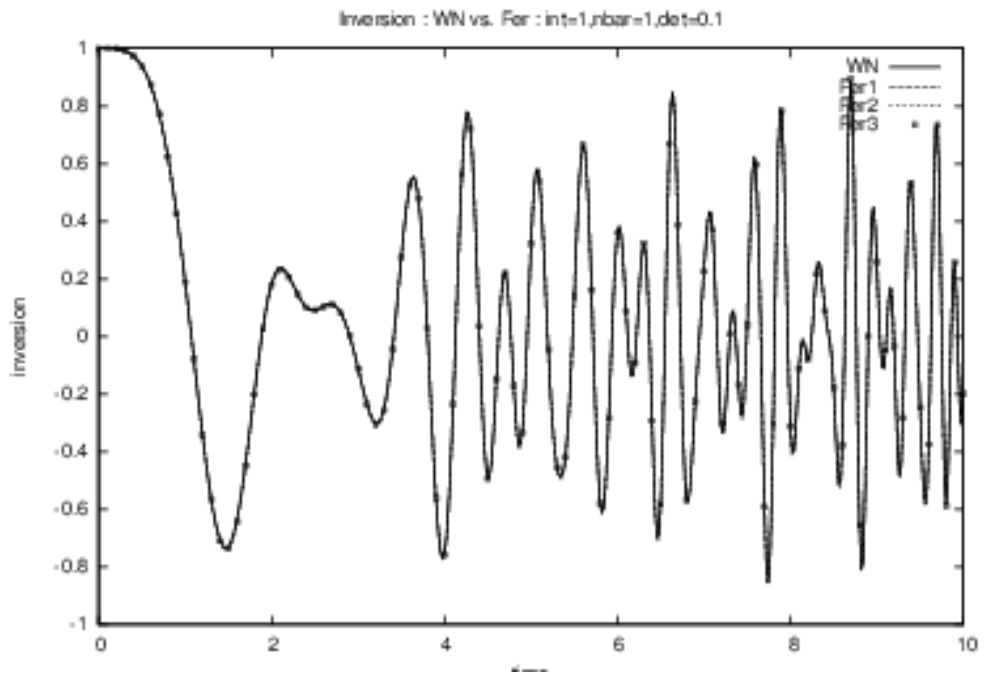

Figure 31: Sweep : Comparing WN and Fer : Variation of inversion.

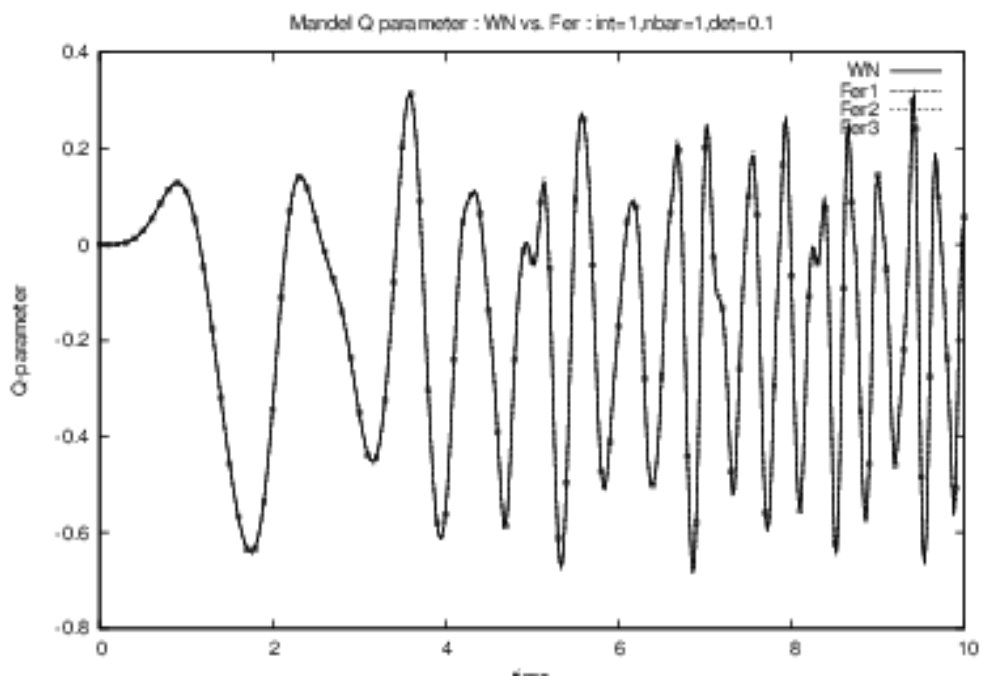

Figure 32: Sweep : Comparing WN and Fer : Variation of Mandel Q parameter.

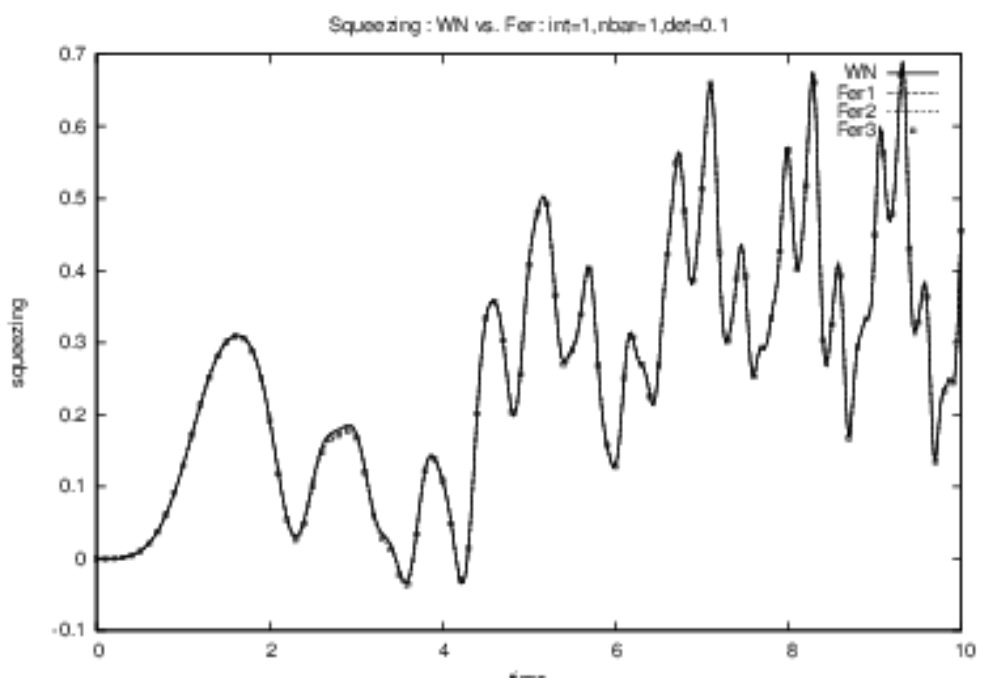

Figure 33: Sweep : Comparing WN and Fer : Variation of squeezing. 


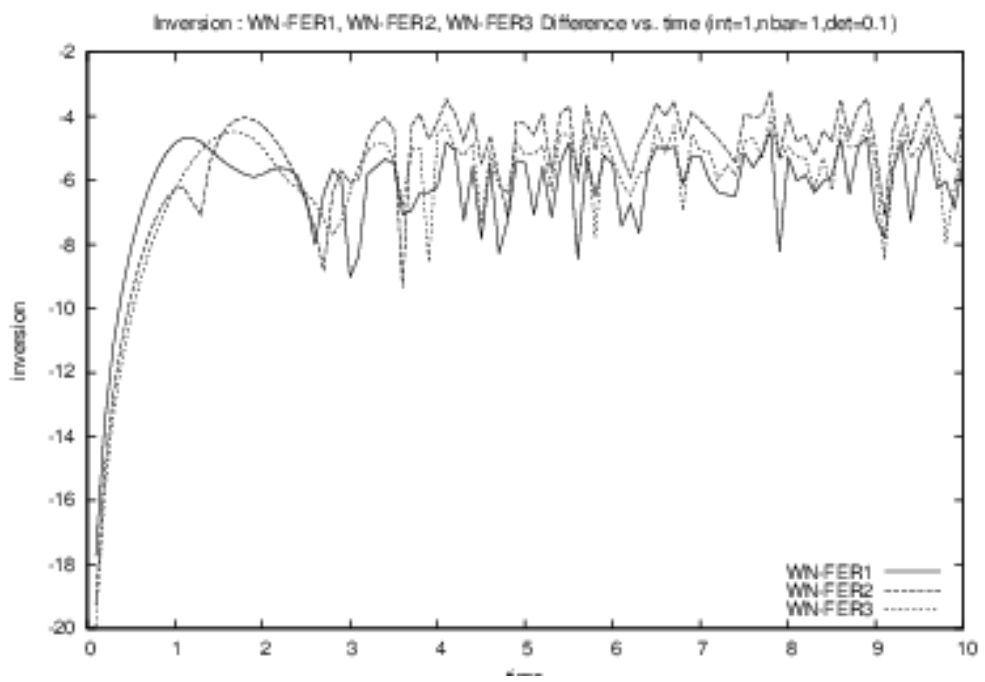

Figure 34: Sweep : Comparing WN and Fer : Variation of inversion : Lorgarithmic Difference.

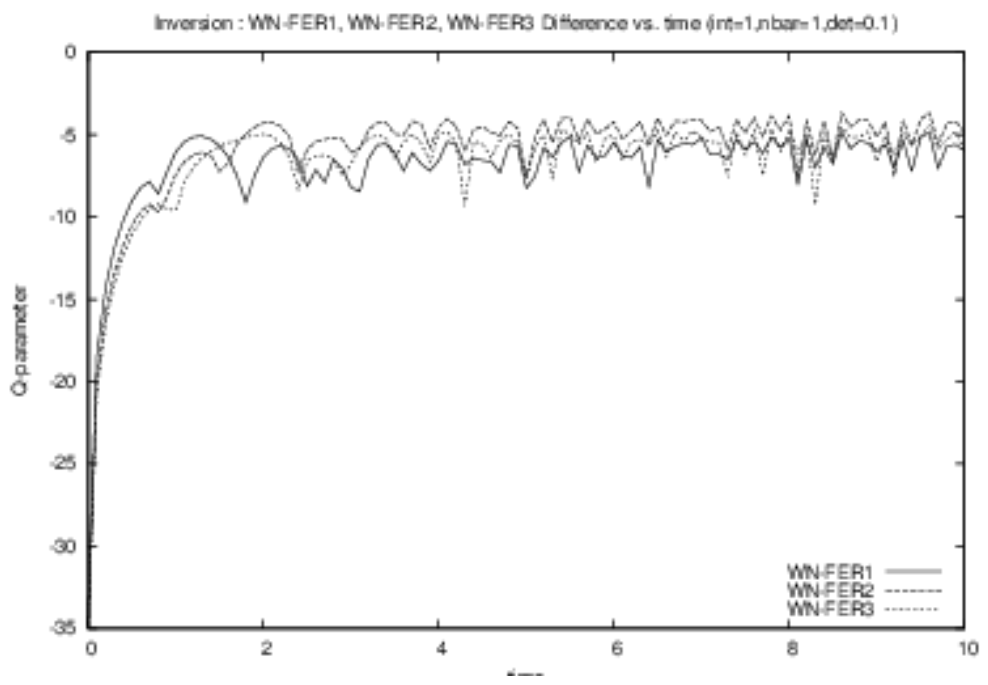

Figure 35: Sweep : Comparing WN and Fer : Variation of Mandel Q parameter : Lorgarithmic Difference.

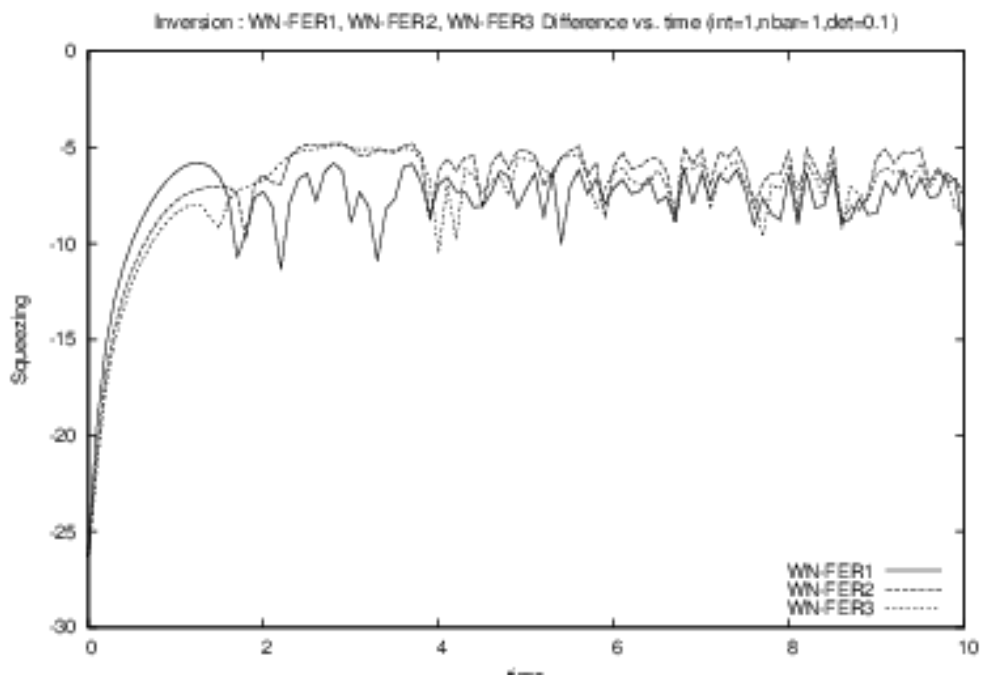

Figure 36: Sweep : Comparing WN and Fer : Variation of squeezing : Lorgarithmic Difference. 


\section{Acknowledgements}

The authors thank Prof. Soumen Kr. Roy (Retired Professor, Jadavpur University, Kolkata, West Bengal, India) for important discussions about Quantum Physics, and are grateful to Indian Association for the Cultivation of Sciences, Kolkata, for providing support to library works.

\section{Appendix : List of integrals}

$$
\begin{array}{lrl}
I_{1}(t)=\int_{0}^{t} d t_{1} \cos \left(\beta t_{1}^{2},\right. & I_{2}(t)=\int_{0}^{t} d t_{1} \sin \left(\beta t_{1}^{2}\right), \\
I_{3}(t)=\int_{0}^{t} d t_{1} \cos \left(\beta t_{1}^{2}\right) I_{1}\left(t_{1}\right), & I_{4}(t)=\int_{0}^{t} d t_{1} \cos \left(\beta t_{1}^{2}\right) I_{2}\left(t_{1}\right), \\
I_{5}(t)=\int_{0}^{t} d t_{1} \sin \left(\beta t_{1}^{2}\right) I_{1}\left(t_{1}\right), & I_{6}(t)=\int_{0}^{t} d t_{1} \sin \left(\beta t_{1}^{2}\right) I_{2}\left(t_{1}\right), \\
I_{7}(t)=\int_{0}^{t} d t_{1} \cos \left(\beta t_{1}^{2}\right) I_{3}\left(t_{1}\right), & I_{8}(t)=\int_{0}^{t} d t_{1} \cos \left(\beta t_{1}^{2}\right) I_{4}\left(t_{1}\right), \\
I_{9}(t)=\int_{0}^{t} d t_{1} \cos \left(\beta t_{1}^{2}\right) I_{5}\left(t_{1}\right), & I_{10}(t)=\int_{0}^{t} d t_{1} \cos \left(\beta t_{1}^{2}\right) I_{6}\left(t_{1}\right), \\
I_{11}(t)=\int_{0}^{t} d t_{1} \sin \left(\beta t_{1}^{2}\right) I_{3}\left(t_{1}\right), & I_{12}(t)=\int_{0}^{t} d t_{1} \sin \left(\beta t_{1}^{2}\right) I_{4}\left(t_{1}\right), \\
I_{13}(t)=\int_{0}^{t} d t_{1} \sin \left(\beta t_{1}^{2}\right) I_{5}\left(t_{1}\right), & I_{14}(t)=\int_{0}^{t} d t_{1} \sin \left(\beta t_{1}^{2}\right) I_{6}\left(t_{1}\right),
\end{array}
$$

\section{References}

[1] W. Magnus, Communications in Pure and Applied Mathematics, $7: 649,1954$.

[2] E.T. Jaynes and F. Cummings, Proc. of the IEEE, 51:89,1963.

[3] W. Wei and E. Norman, J. Math. Phys., 4(4):575,1963.

[4] P. Pechukas and J. C. Light, Journal of Chemical Physics, 44 : 3897, 1966.

[5] R. M. Wilcox, Journal of Mathematical Physics, 8(4 ): 962, 1967.

[6] T.V. Foerster, J. Phys. A : Math. Gen., 8(1):95,1975.

[7] J.H. Eberly, N.B. Narozhny and J.J. Sanchez-Mondragon, Phys. Rev. Lett., 44(20):1323, 1980.

[8] P.L. Knight and P.W. Milonni, Phys. Rep., 66(2):21-107,1980.

[9] H.I. Yoo, J.H. Eberly and J.J. Sanchez-Mondragon, J. Phys. A:Math.Gen., 14:1383,1981.

[10] N.B. Narozhny, J.J. Sanchez-Mondragon and J.H.Eberly, Phys. Rev. A, 23(1):236,1981.

[11] A. Bambini and P.R. Berman, Phys. Rev. A, 23(5):2496,1981.

[12] C. Riti and G. Vetri, Optics Comm., 44(2):105, 1982.

[13] P.L. Knignt and P.M. Radmore, Physics Letters, 90A(7):342,1982. 
[14] S. Klarsfeld and J.A. Oteo, Division de Physique Théorique, IPN, F-91406 Orsay Cedex, France, 1989.

[15] A. Mufti, H. A. Schmitt and M. Sargent III, Americal Journal of Physics, 61 : 729, 1993.

[16] S. Yu, H. Rauch and Y.Zhang, Phys. Rev. A, 52(4):2585,1995.

[17] A. Dasgupta, J. Opt. B : Quantum and Semiclassical Optics, Volume 1, Number 1.

[18] M. Orszag, Quantum Optics, Springer.

[19] A. Dasgupta, Ph.D.Thesis, SINP, Kolkata.

[20] A.B. Klimov and S.M. Chumakov, A Group Theoretical Approach to Quantum Optics, Wiley-VCH.

[21] J.J. Sakurai, Advanced Quantum Mechanics, Second Edition, Addison-Wesley, 1999.

[22] M.Klaiber, D.Dimitrovsky and J.S.Briggs, Physical Review A, 79 : 043402, 2009.

[23] S.Blanes, F.Casas, J.A.Oteo and J.Ros, Physics Reports, 470 : 151-238, 2009. 